\title{
ON PHYSICAL YOGA-THERAPY IN PSYCHIATRY
}

Michailov, M.Ch. 1, Neu, E. 1, Martin, D. 1, Schumitz, H. 1, Schratz, M. 1,2, Weber, G. 1,3, Zöpfl, H. 1,4

1 Inst. Umweltmedizin c/o ICSD/IAS e.V., POB 340316, 80100 Munich, Germany (Int. Council Sci. Develop./Int. Acad. Sci. Berlin-Bratislava-Innsbruck-Muenchen-New Delhi-Paris-Sofia-Vienna)

2 Univ. Innsbruck, School Education Dean, Innsbruck, Austria.

3 Univ. Lxbg. and Vienna, Fac. Psychology Dean, Vienna, Austria.

4 Univ. Muenchen, Fac. Psychol. \& Ped., Inst. Pedag. Dir.a.D., Munich, Germany.

INTRODUCTION. The external respiration is at the same time an autonomic and voluntary physiological process. Through Yoga respiratory training (pranayamas and asanas) there is a possibility of positive influence on some psychopathological processes via feedback mechanisms acc. to psychosomatic (Th.v.UEXKÜLL) and somatopsychic theories (Y.IKEMI) [1].

METHOD. Practises of Indian medicine: Integrative Yoga, Sri YOGENDRA [1+2a]. Psychosomatic practices of occidental/oriental medicine (patients/probands). Evaluation of psychic-"polar-attitude-list"/physiological-parameters: heart-rate, blood-pressure, etc. Integral Yoga, Sri AUROBINDO: Music from Sunil BHATTACHARYA [1+2b].

\section{RESULTS.}

\section{A. Respiratory Therapy (Pranayama)}

A comparative evaluation of some respiratory and cardiovascular parameters of adults practising Hatha-Yoga gave evidence of positive physiological changes. The time of voluntary apnoea after inspiration was significantly prolonged after 1 week training by $21.3 \pm 9.9 \%(37.2 \pm 8.3=>45.2 \pm 10.6 \mathrm{sec} ; n=11, p<0.002)$, after 2 weeks $-52.4 \pm 6.9 \%(36.8 \pm 10.8=>54.0 \pm 21.6 \mathrm{sec} ; n=11, p<0.02)$. The change in time of apnoea after 3 months training in some subjects was extremely high, e.g. 30=>131 sec, 43=>115 sec. The vital capacity increased after 3 months training by $10.9 \pm 7.2 \%(3200 \pm 518 \mathrm{ccm}=>3535 \pm 533 \mathrm{ccm} ; n=10, p<0.02)$. A positive correlation was observed between the respiratory and cardiac changes: the heart rates in normal state and after 10 knee-bendings were reduced (Fig.1).

\section{B. Music, Respiratory, Physical Therapies}

Observations about influence of music [1], respiratory [2], yogaphysical [3] therapies on psychosomatic parameters demonstrate strong positive effects. Items of categories psychophysiological (relaxed), emotional (tranquil/happy), cognitive (few/ordered-thoughts), voluntary (active/spontaneous), social (open/assertive), consciousness (clear/sleepy) are significantly positive changed $25-50 \%$. The 3 therapies have specific psychic effects, e.g. items "relaxed/tranquil" after respiratory-

$(+45 / 50 \%) /$ music-therapy $(+20 / 5 \%)$, also "open" $(+25 \%)$ are positive, but negative after respiratory-therapy $(-20 \%)$. Items are stronger changed before then after gynecological-urologicalsurgical-intervention. Higher effects are observed for investigators and children than of students of psychology. Psychic effects are correlated with positive physiological ones, e.g. heart/respiratory-frequency decreased $25-30 \%$, voluntaryapnoea prolonged 55\% ( $p<0.05-0.01, n=145)$ (Fig. 2-3).

Ref.: [1] Neu, Michailov et al.: Psychiatry: WPA-2019-Lisbon (in press); -2018-Mexico-City (WCP18-0584,-0654,-0643); -2011Buenos-Aires, AB:PO1.200. EPA-2019-Warschau, Eur. Psychiatry 56S, S689; -2018-Nice 48/S1, S636,S567.

Neurology: WFN-2015-Santiago de Chile, J. Neurol. Sci, 357/S1, WFN15-1263,-1273,-1266,-1270; -2019-Dubai (in press). EAN 2015 Berlin, 1st Congr. Eur. Acad. Neurol., Eur.J.Neurol. 22/S1, 487-490,281, 832. Pathology: ESP-2019Nice (in press). [2] a. Sri Yogendra: Hatha-Yoga simplified. The Yoga-Inst. Santa Cruz/Bombay,25/1958 (4th-edition). b. Sri Aurobindo: Integral Yoga. Sri A. Ashram.
CONCLUSION. It is evident that Yoga training could be applied not only for psychosomatic diseases, but also in neurology and psychiatry. There are to be investigated positive effects in patients with neurosis, also depression (stimulation of cerebral exciting processes counteracting to inhibitory ones), etc., see [3] Systematic research in this context could support UNO-Agenda 21 for better health, education, ecology, economy on global level.

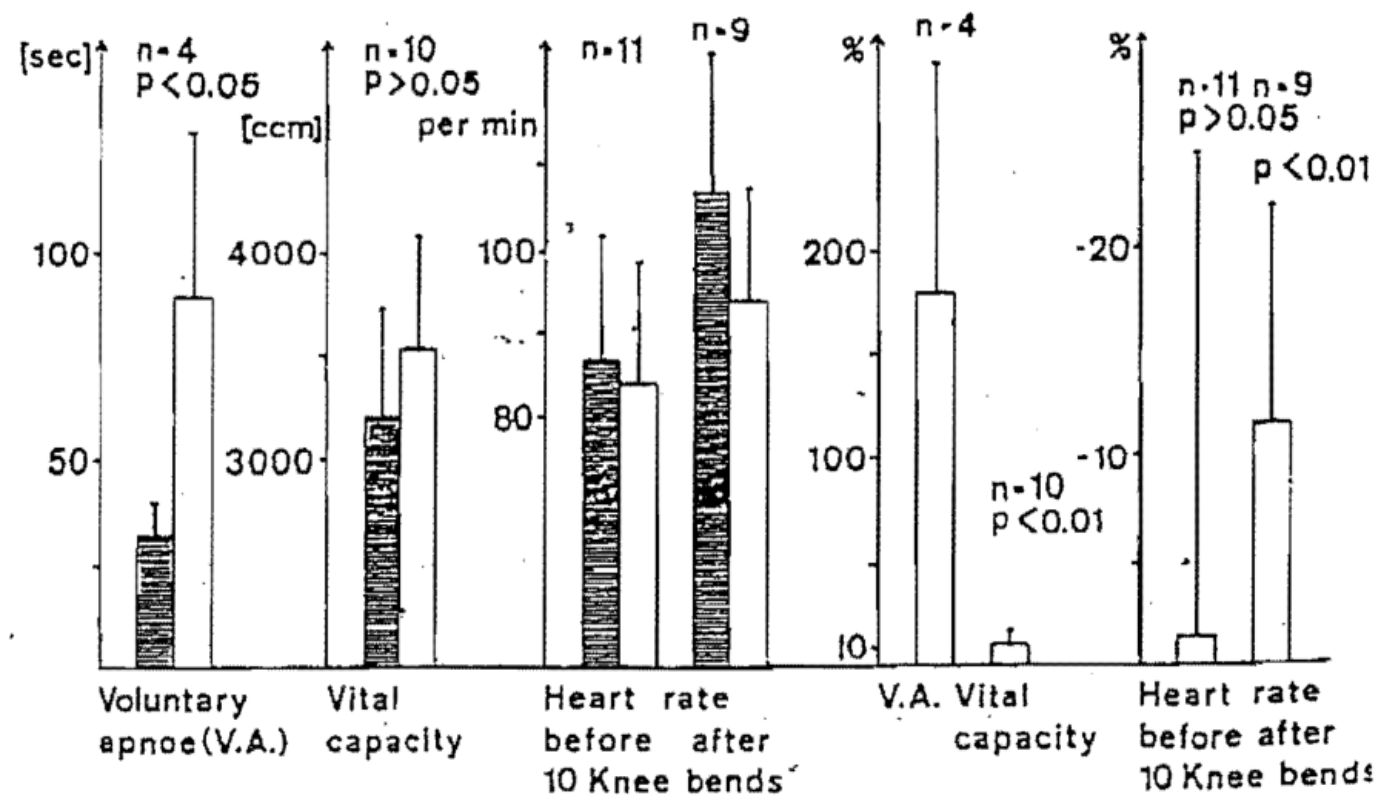

Effects of Hatha-Yoga on some Respiratory and Cardiac Parameters after 3 months

Initial Value

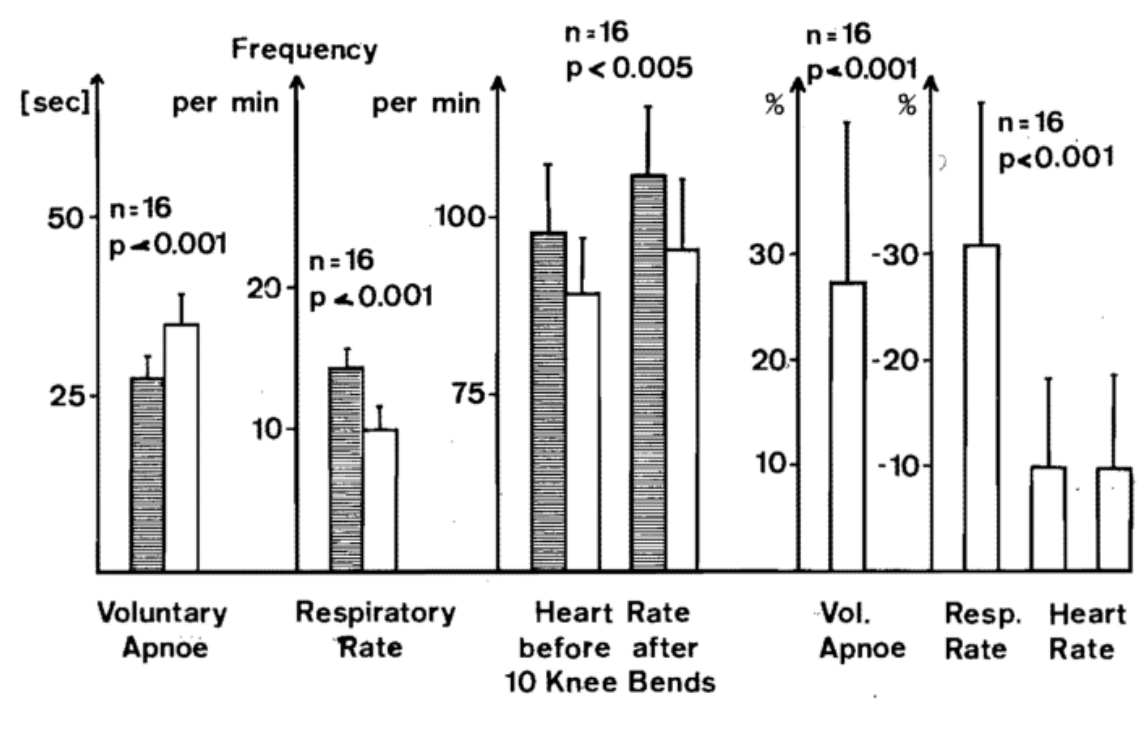
Subject AS: Changes of some Physiological Parameters after each
Hatha - Yoga "Lesson"

寡 Initial Value

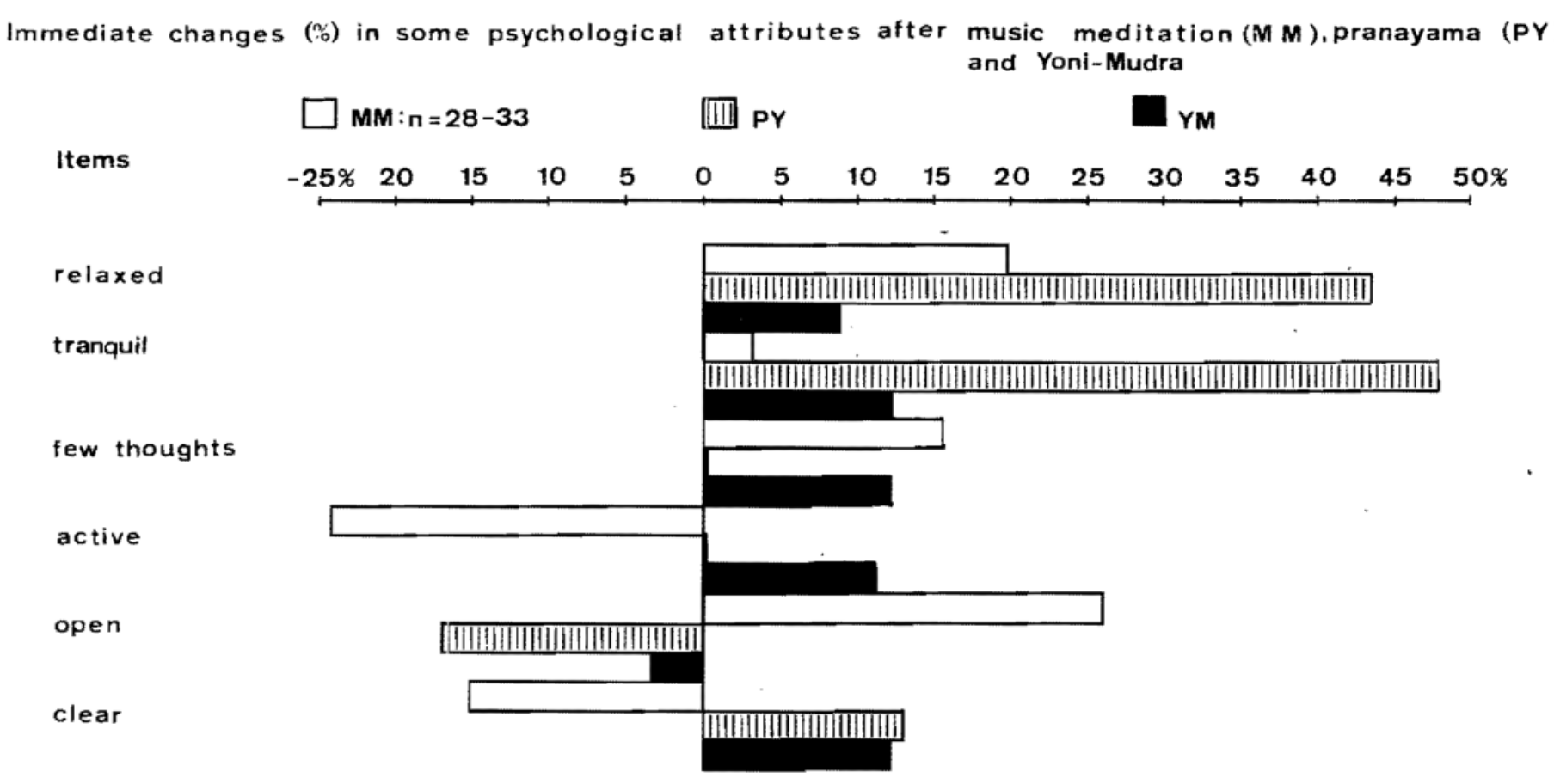

